\title{
ANTIFUNGAL ACTIVITIES OF PRADIMICIN DERIVATIVES MODIFIED AT C4'-AMINO GROUP
}

\author{
Hajime Kamachi, Satsuki Okuyama, Minoru Hirano, Shinji Masuyoshi, \\ Masataka Konishi and ToshiKazU OKI
}

Bristol-Myers Squibb Research Institute, Bristol-Myers Squibb K.K., 2-9-3, Shimo-meguro, Meguro-ku, Tokyo 153, Japan

(Received for publication February 15, 1993)

\begin{abstract}
In order to explore potent derivatives of pradimicins (PRMs), modification of their $\mathrm{C}^{\prime}$-amino group was carried out. 4'- $N$-Cyano $(1,2), 4^{\prime}$-deamino-4'-nitroguanidino (4), 4'-deamino-4'-ureido (7 9) and 4'-deamino-4' -thioureido (10) derivatives were synthesized by trimethylsilylation of PRMs $\mathrm{A}$ and $\mathrm{C}$, followed by condensation with appropriate reagents. $4^{\prime}$-Deamino- $4^{\prime}$-guanidino (5) and $4^{\prime}$-deamino-4'-amidino (6) derivatives were synthesized by catalytic hydrogenation of 4 and 2 , respectively. $4^{\prime}-N$-Nitroso derivative 3 was prepared by treatment of PRM A with nitrous acid. Among these compounds, the 4'-N-cyano derivative of PRM C (2) exhibited in vitro and in vivo antifungal activities comparable to the parent compounds together with good water-solubility.
\end{abstract}

The pradimicins (PRMs) are a new family of antibiotics (Fig. 1) ${ }^{1}{ }^{5}$ that exhibited broad-spectrum antifungal activity both in vitro and in vivo studies. Although they are relatively nontoxic, their limited water-solubility due to amphoteric nature hampered further development studies. Thus, we initiated chemical modification of PRMs focused on the $\mathrm{C}^{\prime}$ - position $^{6 \sim 8)}$ in order to improve water-solubility. In the previous paper $^{8)}$, we reported water-soluble $4^{\prime}-N$-alkyl and -acyl and $4^{\prime}$-deamino- $4^{\prime}$-hydroxy PRM derivatives. This report describes the syntheses and antifungal activities of the other $4^{\prime}$-amino-modified PRM derivatives; $4^{\prime}-N$-cyano, $4^{\prime}-N$-nitroso, $4^{\prime}$-guanidino, $4^{\prime}$-amidino, $4^{\prime}$-ureido and $4^{\prime}$-thioureido derivatives.

\section{Synthesis}

PRMs $\mathrm{A}$ and $\mathrm{C}$ have many functional groups that may interfere with substitution of the $4^{\prime}$-amino group. In the previous paper ${ }^{3)}$, we reported 4 - $N$-alkylation and -acylation of PRMs, with alkyl halides and activated carboxylic acids respectively, proceed smoothly in the presence of $\mathrm{N}, \mathrm{O}$ bis(trimethylsilyl)acetamide (BSA). $N$-Substitution of PRMs with the other reagents also went smoothly. Thus, the BSA-pretreated PRMs A and C were reacted with cyanogen bromide in dichloromethane at room temperature, followed by detrimethylsilylation with $\mathrm{HCl}-\mathrm{MeOH}$ afforded the $4^{\prime}-N$-cyano derivatives, $\mathbf{1}$ and $\mathbf{2}$, respectively, in good yields. The
Fig. 1. Structures of natural pradimicins.

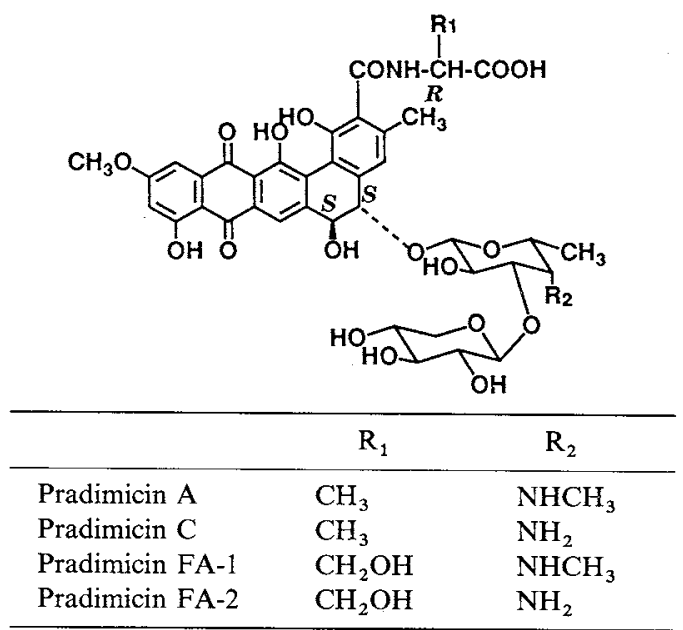

Correspondence should be addressed to Jun OKumura, Britsol-Myers Squibb Research Institute, 2-9-3 Shimomeguro, Meguro-ku, Tokyo 153, Japan. 
Fig. 2. 4'-N-modified pradimicins $(1 \sim 10)$.

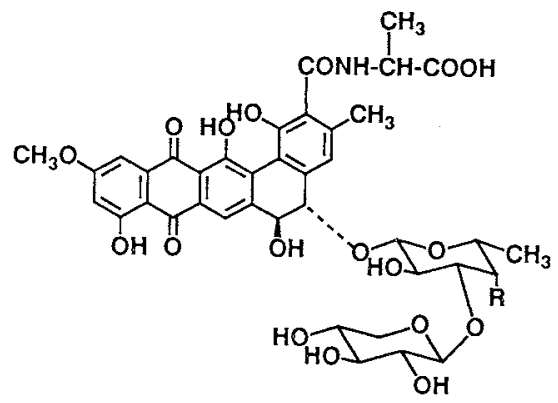

\begin{tabular}{|c|c|}
\hline No. & $\mathbf{R}$ \\
\hline $\mathbf{1}$ & $\mathrm{NCH}_{3} \mathrm{CN}$ \\
\hline 2 & NHCN \\
\hline 3 & $\mathrm{NCH}_{3} \mathrm{NO}$ \\
\hline 4 & $\mathrm{NHC}(=\mathrm{NH}) \mathrm{NHNO}_{2}$ \\
\hline 5 & $\mathrm{NHC}(=\mathrm{NH}) \mathrm{NH}_{2}$ \\
\hline 6 & $\mathrm{NHCH}=\mathrm{NH}$ \\
\hline 7 & $\mathrm{NHCONH}_{2}$ \\
\hline 8 & $\mathrm{NCH}_{3} \mathrm{CONH}_{2}$ \\
\hline 9 & $\mathrm{NCH}_{3} \mathrm{CONHCH}_{3}$ \\
\hline 10 & $\mathrm{NCH}_{3} \mathrm{CSNHCH}_{3}$ \\
\hline
\end{tabular}

Table 1. In vitro activity of pradimicin derivatives

\begin{tabular}{ccccc}
\hline & \multicolumn{4}{c}{ MIC $(\mu \mathrm{g} / \mathrm{ml})^{\mathrm{a}}$} \\
\cline { 2 - 5 } Com- & & & & \\
pound & $\begin{array}{c}\text { Candida } \\
\text { albicans }\end{array}$ & $\begin{array}{c}\text { Candida } \\
\text { tropicalis }\end{array}$ & $\begin{array}{c}\text { Cryptococcus } \\
\text { neoformans }\end{array}$ & $\begin{array}{c}\text { Aspergillus } \\
\text { fumigatus }\end{array}$ \\
& A9540 & IFO 10241 & IAM 4514 & IAM 2034 \\
\hline $\mathbf{1}$ & 3.1 & 12.5 & 3.1 & 12.5 \\
$\mathbf{2}$ & 3.1 & 12.5 & 1.6 & 3.1 \\
$\mathbf{3}$ & 6.3 & 12.5 & 1.6 & 3.1 \\
$\mathbf{4}$ & 3.1 & 12.5 & 1.6 & 3.1 \\
$\mathbf{5}$ & 12.5 & $>100$ & 6.3 & 3.1 \\
$\mathbf{6}$ & 12.5 & $>100$ & 3.1 & 1.6 \\
$\mathbf{7}$ & 6.3 & 12.5 & 6.3 & 50 \\
$\mathbf{8}$ & 12.5 & 25 & 25 & $>100$ \\
$\mathbf{9}$ & 6.3 & 25 & 25 & $>100$ \\
$\mathbf{1 0}$ & 12.5 & 25 & 50 & $>100$ \\
PRM A & 12.5 & $>100$ & 1.6 & 0.8 \\
PRM C & 25 & $>100$ & 0.8 & 3.1 \\
\hline
\end{tabular}

a MIC's were determined by the 2-fold agar dilution method on yeast morphology agar buffered at $\mathrm{pH}$ 7.0 (Incubation, $28^{\circ} \mathrm{C}, 48$ hours).

$N$-unsubstituted cyanoamino derivative 2 was rather unstable in acidic media to give the 4 'ureido derivative 7 , while the $N$-methylcyanoamino derivative 1 was stable in the same conditions. In a similar way to 1 and 2, BSA-pretreated PRM A was reacted with methyl isocyanate and methyl isothiocyanate to afford the 4'-dimethylureido derivative (9) and 4'-dimethylthioureido derivative (10), respectively. PRMs A and $\mathrm{C}$ were reacted with trichloroacetyl isocyanate in a similar way, followed by alkaline hydrolysis, to give the $4^{\prime}$-ureido (7) and the 4'-monomethylureido (8) derivatives, respectively. The reaction of BSA-treated PRM C with $N$-nitro-S-methylisothiourea required more severe conditions. It proceeded in DMF at high temperature $\left(80^{\circ} \mathrm{C}\right.$ for 2 hours) to give the $4^{\prime}$-nitroguanidino derivative 4 , which was subjected to catalytic hydrogenation to afford the $4^{\prime}$-guanidino derivative 5 . Catalytic hydrogenation of the $4^{\prime}$ - $N$-cyano derivative 2 in aqueous acetic acid afforded the $4^{\prime}$-amidino derivative 6 , together with the $4^{\prime}$-ureido derivative 7 , which was considered to be derived from 2 by action of acetic acid. PRM A was treated with sodium nitrite in aqueous acetic acid to give the 4'-nitrosoamino derivative 3 .

\section{In Vitro Activity}

The antifungal activity was determined by the 2-fold agar dilution method on yeast morphology agar buffered with $0.067 \mathrm{M}$ phosphate, $\mathrm{pH} 7.0$ and the results are summarized in Table 1 . The $4^{\prime}-N$-cyano derivative 2 showed improved antifungal activity against Candida albicans and Candida tropicalis and retained antifungal activity of the parent PRM C against Cryptococcus neoformans and Aspergillus fumigatus, while the $4^{\prime}-N$-methylcyano derivative 1 was moderately active against $A$. fumigatus although the activity against the yeasts was very similar to 2 . The 4 '- $N$-nitroso (3) and 4 -nitroguanidino (4) derivatives also showed improved antifungal activity against $C$. tropicalis with retention of activity against all the other strains as compared with PRMs A and C. On the contrary, the 4'-guanidino (5) and 4'-amidino (6) derivatives showed no activity against $C$. tropicalis at $100 \mu \mathrm{g} / \mathrm{ml}$ with retention of the activity against the other strains. The 
4'-ureido derivative 7 was active against only yeasts and the other $N$-substituted ureido or thioureido derivatives $(\mathbf{8} \sim 10)$ showed no activity against A. fumigatus. Among the $\mathrm{C}^{\prime}$ '-amino-modified derivatives, the derivatives 1 through 4 retain in vitro activity of PRMs, but the 4'-ureido and 4'-thioureido derivatives, 7 through 10 , lose activity against A. fumigatus.

In Vivo Activity and Water-solubility of the

\section{4'- $N$-Cyano Derivative 2}

The in vivo activity of $1,2,3$ and 4 , which
Table 2. In vitro activity of pradimicin derivatives against Candida and Aspergillus systemic infections in mice.

\begin{tabular}{ccc}
\hline & \multicolumn{2}{c}{$\mathrm{PD}_{50}(\mathrm{mg} / \mathrm{kg}, \mathrm{iv})$} \\
\cline { 2 - 3 } Compound & $\begin{array}{c}\text { Candida albicans } \\
\text { A9540 }\end{array}$ & $\begin{array}{c}\text { Aspergillus fumigatus } \\
\text { IAM 2034 }\end{array}$ \\
\hline 1 & 13 & $>50$ \\
2 & 13 & 27 \\
$\mathbf{3}$ & 27 & $>50$ \\
$\mathbf{4}$ & 12 & $>50$ \\
PRM A & 10 & 23 \\
PRM C & 13 & $\mathrm{NT}^{2}$ \\
\hline
\end{tabular}

a Not tested. showed broader in vitro antifungal spectrum than PRMs $\mathrm{A}$ and $\mathrm{C}$ was determined in mice infected with $C$. albicans A9540 and A. fumigatus IAM 2034 after intravenous administration, and the results are summarized in Table 2. All of the compounds were as effective as PRMs $\mathrm{A}$ and $\mathrm{C}$ against C. albicans A9540. However, against $A$. fumigatus IAM 2034, only compound 2 was as effective as PRM A among these compounds.

The water solubility of 2 was more than $20 \mathrm{mg} / \mathrm{ml}$ in phosphate-buffered saline containing $\mathrm{Ca}^{2+}$ and $\mathrm{Mg}^{2+}$ at $\mathrm{pH} 7.2^{7}$. Due to the electron withdrawing property of the cyano group, the basicity of 4 -amino group of $\mathbf{2}$ is thought to be much lower than the parent antibiotic resulting in the improvement of its water-solubility at a neutral $\mathrm{pH}$.

In summary, among the 4 -amino-modified derivatives of PRMs A and $\mathrm{C}$ synthesized in this study, the 4 '- $N$-cyano PRM C (2) shows antifungal activity comparable to the PRMs both in vitro and in vivo together with high water-solubility.

\section{Experimental}

MPs were determined using a Yanagimoto micro hot-stage apparatus and are uncorrected. NMR spectra were recorded on a JEOL GX-400 (400 MHz). Mass spectra were recorded on a JEOL JMS-AX505H (FAB) mass spectrometer.

\section{Synthesis}

$4^{\prime}-N$-Cyano Derivatives (1 and 2)

Cyanogen bromide (180 $\mathrm{mg}, 1.7 \mathrm{mmol})$ was added to a mixture of PRM C $(67 \mathrm{mg}, 0.081 \mathrm{mmol})$ and BSA $(0.5 \mathrm{ml}, 2.02 \mathrm{mmol})$ in dichloromethane $(2 \mathrm{ml})$ and the mixture was stirred overnight at room temperature. After removal of the solvent, $\mathrm{MeOH}(2 \mathrm{ml})$ and $1 \mathrm{~N} \mathrm{HCl}(1 \mathrm{ml})$ was added and the mixture was chromatographed on a column of Cosmosil $75 \mathrm{C}_{18}$-OPN (Nacalai Tesque, Inc., $100 \mathrm{~g}$ ). The column was eluted with water and 10 40\% acetonitrile successively. The eluate was collected in fractions, which were monitored by HPLC. The fractions containing 2 were combined, concentrated in vacuo and freeze-dried to give $48 \mathrm{mg}(79 \%)$ of a light red amorphous powder. MP $220 \sim 230^{\circ} \mathrm{C}(\mathrm{dec}) ; \mathrm{IR} v_{\max }(\mathrm{KBr}) \mathrm{cm}^{-1} 2210$, $1720,1620,1290,1060$; Mass (FAB) $m / z 852(\mathrm{M}+\mathrm{H})^{+}$; UV $\lambda_{\max }(1 / 100 \mathrm{~N} \mathrm{NaOH}) \mathrm{nm}(\varepsilon) 319(14,800)$, $496(15,400) ;{ }^{1} \mathrm{H}$ NMR (DMSO- $\left.d_{6}\right) \delta 1.15\left(3 \mathrm{H}, \mathrm{d}, J=7 \mathrm{~Hz}, 5^{\prime}-\mathrm{CH}_{3}\right), 1.33\left(3 \mathrm{H}, \mathrm{d}, J=7 \mathrm{~Hz}, 17-\mathrm{CH}_{3}\right), 2.29$ $\left(3 \mathrm{H}, \mathrm{s}, 3-\mathrm{CH}_{3}\right), 3.93\left(3 \mathrm{H}, \mathrm{s}, \mathrm{OCH}_{3}\right), 4.39(1 \mathrm{H}, \mathrm{q}, J=7 \mathrm{~Hz}, 17-\mathrm{H}), 4.48(1 \mathrm{H}, \mathrm{d}, J=10 \mathrm{~Hz}, 5-\mathrm{H}), 4.52(1 \mathrm{H}$, brd, $J=10 \mathrm{~Hz}, 6-\mathrm{H}), 4.64\left(1 \mathrm{H}, \mathrm{d}, J=8 \mathrm{~Hz}, 1^{\prime}-\mathrm{H}\right), 6.73(1 \mathrm{H}, \mathrm{d}, J=12 \mathrm{~Hz}, \mathrm{NHCN}), 6.87(1 \mathrm{H}, \mathrm{d}, J=2 \mathrm{~Hz}$, $10-\mathrm{H}), 7.11(1 \mathrm{H}, \mathrm{s}, 4-\mathrm{H}), 7.25(1 \mathrm{H}, \mathrm{d}, J=2 \mathrm{~Hz}, 12-\mathrm{H}), 7.93(1 \mathrm{H}, \mathrm{s}, 7-\mathrm{H}), 8.62(1 \mathrm{H}, \mathrm{d}, J=7 \mathrm{~Hz}, 16-\mathrm{NH})$.

Compound 1 was synthesized from PRM A by a similar procedure to the above. Yield $81 \%$; mp $>250^{\circ} \mathrm{C}$ (dec); IR $v_{\max }(\mathrm{KBr}) \mathrm{cm}^{-1} 2200,1720,1600,1290,1165$; Mass (FAB) $m / z 866(\mathrm{M}+\mathrm{H})^{+}$; UV 
$\lambda_{\max }(1 / 100 \mathrm{~N} \mathrm{NaOH}) \mathrm{nm}(\varepsilon) 319(14,800), 496(13,900) ;{ }^{1} \mathrm{H}$ NMR (DMSO- $\left.d_{6}\right) \delta 1.18(3 \mathrm{H}, \mathrm{d}, J=7 \mathrm{~Hz}$, $\left.5^{\prime}-\mathrm{CH}_{3}\right), 1.33\left(3 \mathrm{H}, \mathrm{d}, J=7 \mathrm{~Hz}, 17-\mathrm{CH}_{3}\right), 2.28\left(3 \mathrm{H}, \mathrm{s}, 3-\mathrm{CH}_{3}\right), 3.00\left(3 \mathrm{H}, \mathrm{s}, 4^{\prime}-\mathrm{NCH}_{3}\right), 3.74(1 \mathrm{H}, \mathrm{dd}, J=5$ and $\left.11 \mathrm{~Hz}, 5^{\prime \prime}-\mathrm{H}\right), 3.95\left(3 \mathrm{H}, \mathrm{s}, \mathrm{OCH}_{3}\right), 4.39(1 \mathrm{H}, \mathrm{q}, J=7 \mathrm{~Hz}, 17-\mathrm{H}), 4.41(1 \mathrm{H}, \mathrm{d}, J=10 \mathrm{~Hz}, 5-\mathrm{H}), 4.50(1 \mathrm{H}$, br d, $J=10 \mathrm{~Hz}, 6-\mathrm{H}), 4.64\left(1 \mathrm{H}, \mathrm{d}, J=8 \mathrm{~Hz}, 1^{\prime}-\mathrm{H}\right), 6.89(1 \mathrm{H}, \mathrm{d}, J=2 \mathrm{~Hz}, 10-\mathrm{H}), 7.19(1 \mathrm{H}, \mathrm{s}, 4-\mathrm{H}), 7.26$ $(1 \mathrm{H}, \mathrm{s}, 12-\mathrm{H}), 8.00(1 \mathrm{H}, \mathrm{s}, 7-\mathrm{H}), 8.59(1 \mathrm{H}, \mathrm{s}, 16-\mathrm{NH})$.

\section{$4^{\prime}-N$-Nitroso Derivative (3)}

Sodium nitrite (1 $\mathrm{M}$ aqueous solution, $0.5 \mathrm{ml}$ ) was added dropwise to a stirred solution of PRM A $(100 \mathrm{mg}, 1.19 \mathrm{mmol})$ in $0.25 \mathrm{M}$ aqueous acetic acid $(10 \mathrm{ml})$. The mixture was stirred for 2 hours at room temperature. By a similar purification to $2,75 \mathrm{mg}(72 \%)$ of 3 was obtained. MP $230 \sim 240^{\circ} \mathrm{C}$ (dec); IR $v_{\max }(\mathrm{KBr}) \mathrm{cm}^{-1} 1720,1600,1450,1295,1160,1060 ;$ Mass $(\mathrm{FAB}) \mathrm{m} / \mathrm{z} 870(\mathrm{M}+\mathrm{H})^{+} ; \mathrm{UV} \lambda_{\max }(1 / 100 \mathrm{~N}$ $\mathrm{NaOH}) \mathrm{nm}(\varepsilon) 320(14,100), 498(14,000) ;{ }^{1} \mathrm{H}$ NMR $\left(\mathrm{DMSO}-d_{6}\right) \delta 0.98\left(3 \mathrm{H}, \mathrm{d}, J=7 \mathrm{~Hz}, 5^{\prime}-\mathrm{CH}_{3}\right), 1.33$ $\left(3 \mathrm{H}, \mathrm{d}, J=7 \mathrm{~Hz}, 17-\mathrm{CH}_{3}\right), 2.29\left(3 \mathrm{H}, \mathrm{s}, 3-\mathrm{CH}_{3}\right), 3.15\left(3 \mathrm{H}, \mathrm{s}, 4^{\prime}-\mathrm{NCH}_{3}\right), 3.67(1 \mathrm{H}, \mathrm{dd}, J=5$ and $11 \mathrm{~Hz}$, $\left.5^{\prime \prime}-\mathrm{H}\right), 3.95\left(3 \mathrm{H}, \mathrm{s}, \mathrm{OCH}_{3}\right), 4.40(1 \mathrm{H}, \mathrm{q}, J=7 \mathrm{~Hz}, 17-\mathrm{H}), 4.47\left(1 \mathrm{H}, \mathrm{d}, J=7 \mathrm{~Hz}, \mathrm{l}^{\prime \prime}-\mathrm{H}\right), 4.54(2 \mathrm{H}, \mathrm{br} \mathrm{s}, 5-\mathrm{H}$ and $6-\mathrm{H}), 4.81\left(1 \mathrm{H}, \mathrm{d}, J=8 \mathrm{~Hz}, 1^{\prime}-\mathrm{H}\right), 6.92(1 \mathrm{H}, \mathrm{d}, J=2 \mathrm{~Hz}, 10-\mathrm{H}), 7.04(1 \mathrm{H}, \mathrm{s}, 4-\mathrm{H}), 7.28(1 \mathrm{H}, \mathrm{d}, J=2 \mathrm{~Hz}$, $12-\mathrm{H}), 8.20(1 \mathrm{H}, \mathrm{s}, 7-\mathrm{H}), 8.59(1 \mathrm{H}, \mathrm{d}, J=7 \mathrm{~Hz}, 16-\mathrm{NH})$.

\section{4'-Nitroguanidino Derivative (4)}

$N$-Nitro- $S$-methylisothiourea $(150 \mathrm{mg}, 1.14 \mathrm{mmol})$ was added to a mixture of PRM A $(100 \mathrm{mg}$, $1.21 \mathrm{mmol})$ and BSA $(0.5 \mathrm{ml}, 2.02 \mathrm{mmol})$ in DMF $(2 \mathrm{ml})$ and the mixture was heated at $80^{\circ} \mathrm{C}$ for 2 hours. After removal of the solvent, $\mathrm{MeOH}(2 \mathrm{ml})$ and $1 \mathrm{~N} \mathrm{HCl}(1 \mathrm{ml})$ was added and the mixture was purified by a similar way to that of compound 2 to give $69 \mathrm{mg}(63 \%)$ of 4 . MP $220 \sim 230^{\circ} \mathrm{C}(\mathrm{dec}) ; \mathrm{IR} v_{\max }(\mathrm{KBr})$ $\mathrm{cm}^{-1} 1720,1600,1290,1160,1050$; Mass (FAB) $m / z 914(\mathrm{M}+\mathrm{H})^{+}$; UV $\lambda_{\max }(1 / 100 \mathrm{~N} \mathrm{NaOH}) \mathrm{nm}(\varepsilon) 316$ $(16,000), 497(14,700) ;{ }^{1} \mathrm{H}$ NMR (DMSO- $\left.d_{6}\right) \delta 1.06\left(3 \mathrm{H}, \mathrm{d}, J=7 \mathrm{~Hz}, 5^{\prime}-\mathrm{CH}_{3}\right), 1.34(3 \mathrm{H}, \mathrm{d}, J=7 \mathrm{~Hz}$, $\left.17-\mathrm{CH}_{3}\right), 2.32\left(3 \mathrm{H}, \mathrm{s}, 3-\mathrm{CH}_{3}\right), 3.95\left(3 \mathrm{H}, \mathrm{s}, \mathrm{OCH}_{3}\right), 4.40(1 \mathrm{H}, \mathrm{q}, J=7 \mathrm{~Hz}, 17-\mathrm{H}), 4.44(1 \mathrm{H}, \mathrm{d}, J=7 \mathrm{~Hz}$, $\left.1^{\prime \prime}-\mathrm{H}\right), 4.50(1 \mathrm{H}, \mathrm{d}, J=10 \mathrm{~Hz}, 5-\mathrm{H}), 4.60(1 \mathrm{H}, \mathrm{brd}, J=10 \mathrm{~Hz}, 6-\mathrm{H}), 4.76\left(1 \mathrm{H}, \mathrm{d}, J=8 \mathrm{~Hz}, 1^{\prime}-\mathrm{H}\right), 6.91(1 \mathrm{H}$, $\mathrm{s}, 10-\mathrm{H}), 7.11(1 \mathrm{H}, \mathrm{s}, 4-\mathrm{H}), 7.28(1 \mathrm{H}, \mathrm{s}, 12-\mathrm{H}), 8.02(1 \mathrm{H}, \mathrm{s}, 7-\mathrm{H}), 8.59(1 \mathrm{H}, \mathrm{d}, J=7 \mathrm{~Hz}, 16-\mathrm{NH})$.

\section{4'-Guanidino PRM C (5)}

A mixture of $4(50 \mathrm{mg}, 0.055 \mathrm{mmol})$ and $10 \%$ palladium on charcoal $(20 \mathrm{mg})$ in $1 \mathrm{~N} \mathrm{HCl}-\mathrm{MeOH}(1: 10$, $5 \mathrm{ml}$ ) was hydrogenated overnight under atmospheric pressure. The mixture was chromatographed on a column of Cosmosil $75 \mathrm{C}_{18}$-OPN (Nacalai Tesque, Inc., $100 \mathrm{~g}$ ) eluting with water and then with 1/1,000 N hydrochloric acid - acetonitrile $(80: 20 \sim 60: 40)$ successively. Concentration of the appropriate fractions gave $18 \mathrm{mg}(38 \%)$ of 5 . MP $220 \sim 230^{\circ} \mathrm{C}(\mathrm{dec})$; IR $v_{\max }(\mathrm{KBr}) \mathrm{cm}^{-1} 1720,1605,1290,1150$; Mass (FAB) $\mathrm{m} / \mathrm{z}$ $869(\mathrm{M}+\mathrm{H})^{+} ; \mathrm{UV} \lambda_{\max }(1 / 100 \mathrm{~N} \mathrm{NaOH}) \mathrm{nm}(\varepsilon) 318(14,700), 498(14,100) ;{ }^{1} \mathrm{H}$ NMR (DMSO- $\left.d_{6}\right) \delta 1.08$ $\left(3 \mathrm{H}, \mathrm{d}, J=7 \mathrm{~Hz}, 5^{\prime}-\mathrm{CH}_{3}\right), 1.34\left(3 \mathrm{H}, \mathrm{d}, J=7 \mathrm{~Hz}, 17-\mathrm{CH}_{3}\right), 2.30\left(3 \mathrm{H}, \mathrm{s}, 3-\mathrm{CH}_{3}\right), 3.95\left(3 \mathrm{H}, \mathrm{s}, \mathrm{OCH}_{3}\right), 4.40$ $(1 \mathrm{H}, \mathrm{q}, J=7 \mathrm{~Hz}, 17-\mathrm{H}), 4.43\left(1 \mathrm{H}, \mathrm{d}, J=7 \mathrm{~Hz}, 1^{\prime \prime}-\mathrm{H}\right), 4.55(2 \mathrm{H}, \mathrm{brs}, 5-\mathrm{H}$ and $6-\mathrm{H}), 4.76(1 \mathrm{H}, \mathrm{d}, J=8 \mathrm{~Hz}$, $\left.1^{\prime}-\mathrm{H}\right), 6.85(1 \mathrm{H}, \mathrm{s}, 10-\mathrm{H}), 7.04(1 \mathrm{H}, \mathrm{s}, 4-\mathrm{H}), 7.24(1 \mathrm{H}, \mathrm{s}, 12-\mathrm{H}), 7.88(1 \mathrm{H}, \mathrm{s}, 7-\mathrm{H}), 8.65(1 \mathrm{H}, \mathrm{d}, J=7 \mathrm{~Hz}, 16-\mathrm{NH})$.

\section{4'-Amidino PRM C (6)}

A mixture of $2(35 \mathrm{mg}, 0.041 \mathrm{mmol})$ and $10 \%$ palladium on charcoal $(10 \mathrm{mg})$ in $25 \%$ aqueous acetic acid was hydrogenated overnight under atmospheric pressure. HPLC showed the presence of two products. Chromatographic separation by Cosmosil $75 \mathrm{C}_{18}$-OPN column (100 g) eluting with $10 \sim 40 \%$ acetonitrile afforded $10 \mathrm{mg}(27 \%)$ of 7 . Further elution with $1 / 1,000 \mathrm{~N} \mathrm{HCl}$-acetonitrile $(80: 20 \sim 60: 40)$ afforded $5 \mathrm{mg}$ (14\%) of 6. MP $220 \sim 230^{\circ} \mathrm{C}(\mathrm{dec})$; IR $v_{\max }(\mathrm{KBr}) \mathrm{cm}^{-1} 1700,1605,1290,1160$; Mass (FAB) $\mathrm{m} / \mathrm{z} 854$ $(\mathrm{M}+\mathrm{H})^{+} ; \mathrm{UV} \lambda_{\max } 1 / 100 \mathrm{~N} \mathrm{NaOH} \mathrm{nm}(\varepsilon) 319(14,100), 496(14,900) ;{ }^{1} \mathrm{H}$ NMR (DMSO- $\left.d_{6}\right) \delta 1.09(3 \mathrm{H}$, d, $\left.J=7 \mathrm{~Hz}, 5^{\prime}-\mathrm{CH}_{3}\right), 1.34\left(3 \mathrm{H}, \mathrm{d}, J=7 \mathrm{~Hz}, 17-\mathrm{CH}_{3}\right), 2.29\left(3 \mathrm{H}, \mathrm{s}, 3-\mathrm{CH}_{3}\right), 3.94\left(3 \mathrm{H}, \mathrm{s}, \mathrm{OCH}_{3}\right), 4.39(1 \mathrm{H}$, q, $J=7 \mathrm{~Hz}, 17-\mathrm{H}), 4.55(2 \mathrm{H}, \mathrm{br}, 5-\mathrm{H}$ and $6-\mathrm{H}), 6.83(1 \mathrm{H}, \mathrm{s}, 10-\mathrm{H}), 7.02(1 \mathrm{H}, \mathrm{s}, 4-\mathrm{H}), 7.22(1 \mathrm{H}, \mathrm{s}, 12-\mathrm{H})$, $7.82(1 \mathrm{H}, \mathrm{s}, 7-\mathrm{H}), 7.90(1 \mathrm{H}, \mathrm{s}, \mathrm{NHCH}=\mathrm{NH}), 8.68(1 \mathrm{H}, \mathrm{br}, 16-\mathrm{NH})$.

\section{4'-Ureido PRMs (7 9) and 4'-Thioureido PRM (10)}

Trichloroacetyl isocyanate ( $316 \mathrm{mg}, 1.7 \mathrm{mmol}$ ) was added to a mixture of PRM C ( $85 \mathrm{mg}, 0.102 \mathrm{mmol})$ and BSA $(0.5 \mathrm{ml}, 2.02 \mathrm{mmol})$ in dichloromethane $(2 \mathrm{ml})$ and the mixture was stirred overnight at room 
temperature. After removal of the solvent, $\mathrm{MeOH}(2 \mathrm{ml})$ and $1 \mathrm{~N} \mathrm{HCl}(1 \mathrm{ml})$ was added. Separation of the mixture by Cosmosil $75 \mathrm{C}_{18}$ - $\mathrm{OPN}$ column with $40 \%$ acetonitrile elution afforded $\mathrm{N}$-trichloroacetyl ureido derivative $(80 \mathrm{mg})$. This was dissolved in $1 \mathrm{~N} \mathrm{NaOH}(1 \mathrm{ml})$ and stirred for 1 hour at room temperature. The solution was acidified and chromatographed on a column of Cosmosil $75 \mathrm{C}_{18}$-OPN. Elution with $20 \%$ acetonitrile afforded $47 \mathrm{mg}(53 \%)$ of $7 . \mathrm{MP} 220 \sim 230^{\circ} \mathrm{C}(\mathrm{dec})$; IR $v_{\max }(\mathrm{KBr}) \mathrm{cm}^{-1} 1600,1300,1050$; Mass (FAB) $m / z 870(\mathrm{M}+\mathrm{H})^{+} ; \mathrm{UV} \lambda_{\max }(1 / 100 \mathrm{~N} \mathrm{NaOH}) \mathrm{nm}(\varepsilon) 319(14,900), 498(14,900) ;{ }^{1} \mathrm{H}$ NMR $\left(\mathrm{DMSO}-d_{6}\right)$ $\delta 1.00\left(3 \mathrm{H}, \mathrm{d}, J=7 \mathrm{~Hz}, 5^{\prime}-\mathrm{CH}_{3}\right), 1.34\left(3 \mathrm{H}, \mathrm{d}, J=7 \mathrm{~Hz}, 17-\mathrm{CH}_{3}\right), 2.31\left(3 \mathrm{H}, \mathrm{s}, 3-\mathrm{CH}_{3}\right), 3.95\left(3 \mathrm{H}, \mathrm{s}, \mathrm{OCH}_{3}\right)$, $4.39(1 \mathrm{H}, \mathrm{q}, J=7 \mathrm{~Hz}, 17-\mathrm{H}), 4.42\left(1 \mathrm{H}, \mathrm{d}, J=7 \mathrm{~Hz}, 1^{\prime \prime}-\mathrm{H}\right), 4.53(2 \mathrm{H}, \mathrm{s}, 5-\mathrm{H}$ and $6-\mathrm{H}), 4.66(1 \mathrm{H}, \mathrm{d}, J=8 \mathrm{~Hz}$, $\left.\mathrm{l}^{\prime}-\mathrm{H}\right), 6.91(1 \mathrm{H}, \mathrm{d}, J=2 \mathrm{~Hz}, 10-\mathrm{H}), 7.12(1 \mathrm{H}, \mathrm{s}, 4-\mathrm{H}), 7.27(1 \mathrm{H}, \mathrm{d}, J=2 \mathrm{~Hz}, 12-\mathrm{H}), 7.98(1 \mathrm{H}, \mathrm{s}, 7-\mathrm{H}), 8.61$ $(1 \mathrm{H}, \mathrm{d}, J=7 \mathrm{~Hz}, 16-\mathrm{NH})$.

Compounds 8 was synthesized from PRM A by a similar procedure to above. Yield $50 \%$; MP $215 \sim 235^{\circ} \mathrm{C}(\mathrm{dec}) ; \mathrm{IR} v_{\max }(\mathrm{KBr}) \mathrm{cm}^{-1} 1600,1300,1060$; Mass (FAB) $m / z 884(\mathrm{M}+\mathrm{H})^{+}$; UV $v_{\max }(1 / 100 \mathrm{~N}$ $\mathrm{NaOH}) \mathrm{nm}(\varepsilon) 317(13,900), 498(13,300) ;{ }^{1} \mathrm{H}$ NMR $\left(\mathrm{DMSO}-d_{6}\right) \delta 1.02\left(3 \mathrm{H}, \mathrm{d}, J=7 \mathrm{~Hz}, 5^{\prime}-\mathrm{CH}_{3}\right), 1.33$ $\left(3 \mathrm{H}, \mathrm{d}, J=7 \mathrm{~Hz}, 17-\mathrm{CH}_{3}\right), 2.29\left(3 \mathrm{H}, \mathrm{s}, 3-\mathrm{CH}_{3}\right), 3.02\left(3 \mathrm{H}, \mathrm{s}, \mathrm{N}-\mathrm{CH}_{3}\right), 3.95\left(3 \mathrm{H}, \mathrm{s}, \mathrm{OCH}_{3}\right), 4.39(1 \mathrm{H}, \mathrm{q}$, $J=7 \mathrm{~Hz}, 17-\mathrm{H}), 4.45(1 \mathrm{H}, \mathrm{d}, J=11 \mathrm{~Hz}, 5-\mathrm{H}), 4.51\left(2 \mathrm{H}, \mathrm{m}, 6-\mathrm{H}\right.$ and $\left.1^{\prime \prime}-\mathrm{H}\right), 4.67\left(1 \mathrm{H}, \mathrm{d}, J=8 \mathrm{~Hz}, 1^{\prime}-\mathrm{H}\right)$, $6.87(1 \mathrm{H}, \mathrm{d}, J=2 \mathrm{~Hz}, 10-\mathrm{H}), 7.00(1 \mathrm{H}, \mathrm{s}, 4-\mathrm{H}), 7.24(1 \mathrm{H}, \mathrm{d}, J=2 \mathrm{~Hz}, 12-\mathrm{H}), 7.95(1 \mathrm{H}, \mathrm{s}, 7-\mathrm{H}), 8.65(1 \mathrm{H}$, d, $J=7 \mathrm{~Hz}, 16-\mathrm{NH})$.

Compound 9 and 10 were synthesized by coupling of PRM A with methyl isocyanate and methyl isothiocyanate, respectively, in a similar reaction conditions to above. 9; yield $52 \%$; MP $210 \sim 230^{\circ} \mathrm{C}(\mathrm{dec})$; IR $v_{\max }(\mathrm{KBr}) \mathrm{cm}^{-1} 1600,1300,1060$; Mass $(\mathrm{FAB}) \mathrm{m} / \mathrm{z} 897(\mathrm{M}+\mathrm{H})^{-}$; UV $\lambda_{\max }(1 / 100 \mathrm{~N} \mathrm{NaOH}) \mathrm{nm}(\varepsilon)$ $319(14,700), 498(14,400) ;{ }^{1} \mathrm{H}$ NMR $\left(\mathrm{DMSO}-d_{6}\right) \delta 1.01\left(3 \mathrm{H}, \mathrm{d}, J=7 \mathrm{~Hz}, 5^{\prime}-\mathrm{CH}_{3}\right), 1.33(3 \mathrm{H}, \mathrm{d}, J=7 \mathrm{~Hz}$, $\left.17-\mathrm{CH}_{3}\right), 2.28\left(3 \mathrm{H}, \mathrm{s}, 3-\mathrm{CH}_{3}\right), 2.55\left(3 \mathrm{H}, \mathrm{d}, J=5 \mathrm{~Hz}, \mathrm{NHCH}_{3}\right), 3.02\left(3 \mathrm{H}, \mathrm{s}, \mathrm{N}-\mathrm{CH}_{3}\right), 3.95\left(3 \mathrm{H}, \mathrm{s}, \mathrm{OCH}_{3}\right)$, $4.39(1 \mathrm{H}, \mathrm{q}, J=7 \mathrm{~Hz}, 17-\mathrm{H}), 4.45(1 \mathrm{H}, \mathrm{d}, J=11 \mathrm{~Hz}, 5-\mathrm{H}), 4.51\left(2 \mathrm{H}, \mathrm{m}, 6-\mathrm{H}\right.$ and $\left.1^{\prime \prime}-\mathrm{H}\right), 4.68(1 \mathrm{H}, \mathrm{d}, J=8 \mathrm{~Hz}$, $\left.1^{\prime}-\mathrm{H}\right), 6.84(1 \mathrm{H}, \mathrm{d}, J=2 \mathrm{~Hz}, 10-\mathrm{H}), 6.99(1 \mathrm{H}, \mathrm{s}, 4-\mathrm{H}), 7.22(1 \mathrm{H}, \mathrm{d}, J=2 \mathrm{~Hz}, 12-\mathrm{H}), 7.91(1 \mathrm{H}, \mathrm{s}, 7-\mathrm{H}), 8.70$ $(1 \mathrm{H}, \mathrm{d}, J=7 \mathrm{~Hz}, 16-\mathrm{NH}) .10$; yield $76 \%$; MP $230 \sim 235^{\circ} \mathrm{C}$ (dec); IR $v_{\max }(\mathrm{KBr}) \mathrm{cm}^{-1} 1600,1300,1060$; Mass (FAB) $m / z 914(\mathrm{M}+\mathrm{H})^{-}$; UV $\lambda_{\max }(1 / 100 \mathrm{~N} \mathrm{NaOH}) \mathrm{nm}(\varepsilon) 319(16,600), 498(15,900) ;{ }^{1} \mathrm{H} \mathrm{NMR}$ $\left(\mathrm{DMSO}_{6}\right) \delta 1.05\left(3 \mathrm{H}, \mathrm{d}, J=7 \mathrm{~Hz}, 5^{\prime}-\mathrm{CH}_{3}\right), 1.37\left(3 \mathrm{H}, \mathrm{d}, J=7 \mathrm{~Hz}, 17-\mathrm{CH}_{3}\right), 2.29\left(3 \mathrm{H}, \mathrm{s}, 3-\mathrm{CH}_{3}\right), 2.90$ $\left(3 \mathrm{H}, \mathrm{d}, J=4 \mathrm{~Hz}, \mathrm{NHCH}_{3}\right), 3.94\left(3 \mathrm{H}, \mathrm{s}, \mathrm{OCH}_{3}\right), 4.39(1 \mathrm{H}, \mathrm{q}, J=7 \mathrm{~Hz}, 17-\mathrm{H}), 4.47(1 \mathrm{H}, \mathrm{d}, J=11 \mathrm{~Hz}, 5-\mathrm{H})$, $4.52\left(2 \mathrm{H}, \mathrm{m}, 6-\mathrm{H}\right.$ and $\left.\mathrm{l}^{\prime \prime}-\mathrm{H}\right), 4.70\left(1 \mathrm{H}, \mathrm{d}, J=8 \mathrm{~Hz}, \mathrm{l}^{\prime}-\mathrm{H}\right), 6.91(1 \mathrm{H}, \mathrm{d}, J=2 \mathrm{~Hz}, 10-\mathrm{H}), 7.03(1 \mathrm{H}, \mathrm{s}, 4-\mathrm{H})$, $7.27(1 \mathrm{H}, \mathrm{d}, J=2 \mathrm{~Hz}, 12-\mathrm{H}), 8.00(1 \mathrm{H}, \mathrm{s}, 7-\mathrm{H}), 8.62(1 \mathrm{H}, \mathrm{d}, J=7 \mathrm{~Hz}, 16-\mathrm{NH})$.

\section{Susceptibility Testing}

MICs were determined on yeasts morphology agar (YMA, Difco Laboratories, Detroit, Mich., U.S.A.) buffered with $0.067 \mathrm{~m}$ phosphate, $\mathrm{pH} 7.0$. Nine parts of molten agar were combined with one part of antibiotic dilution petri dishes. A 5- $\mu$ l suspension containing $2 \times 10^{6}$ cells per $\mathrm{ml}$ was spotted on the surface of the agar plates. The plates were incubated at $28^{\circ} \mathrm{C}$ for 60 hours. MICs were recorded after 40 hours of incubation and defined as the lowest antibiotic concentrations showing no growth or less than five discrete colonies per spot.

\section{Experimental Infection in Mice}

Groups of 5 male ICR mice weighing $20 \sim 24 \mathrm{~g}$ at each dose level received $10-\mathrm{LD}_{50}$ of $C$. albicans A9540 or A. fumigatus IAM 2034 intravenously and test compounds given intravenously once immediately after the infection. The $50 \%$ protective dose $\left(\mathrm{PD}_{50}\right)$ was calculated by the method of LITCHFIELD and WILCOXON $^{9}$ from the survival rate 20 days after the fungal infection.

\section{Acknowledgment}

The authors wish to thank Dr. T. Furumal and Mr. K. SAITOH for the supply of the fermentation products.

\section{References}

1) OKI, T.; M. Konishi, K. Tomatsu, K. Tomita, K. Saitoh, M. Tsunakawa, M. Nishio, T. Miyaki \& H. Kawaguchi: Pradimicin, a novel class of potent antifungal antibiotics. J. Antibiotics 41: $1701 \sim 1704,1988$

2) Tsunakawa, M.; M. Nishio, H. Ohkuma, T. Tsuno, M. Konishi, T. Naito, T. Oki \& H. Kawaguchi: The 
structures of pradimicins A, B and C, a novel family of antifungal antibiotics. J. Org. Chem. 54: 2532 2536, 1989

3) Tomita, K.; M. Nishio, K. Saitoh, H. Yamamoto, Y. Hoshino, H. Ohkuma, M. Konishi, T. MiYaKi \& T. OKi: Pradimicins A, B and C: New antifungal antibiotics. I. Taxonomy, production, isolation and physico-chemical properties. J. Antibiotics 43: 755 762, 1990

4) OKI, T.; O. Tenmyo, M. Hirano, K. Tomatsu \& H. Kamel: Pradimicins A, B and C: New antifungal antibiotics. II. In vitro and in vivo biological activities. J. Antibiotics 43: 763 770, 1990

5) Sawada, Y.; M. Hatori, H. Yamamoto, M. Nisho, T. Miyaki \& T. OKI: New antifungal antibiotics pradimicins FA-1 and FA-2: D-Serine analogs of pradimicins A and C. J. Antibiotics 43: $1223 \sim 1229,1990$

6) OKi, T.; M. Kakushima, M. Nishio, H. Kamei, M. HiRano, Y. Sawada \& M. Konishi: Water-soluble pradimicin derivatives, synthesis and antifungal evaluation of $N, N$-dimethyl pradimicins. J. Antibiotics 43: 1230 1235, 1990

7) Kakushima, M.; S. Masuyoshi, M. Hirano, M. Shinoda, A. Ohta, H. Kamei \& T. Oki: In vitro and in vivo antifungal activities of BMY-28864, a water-soluble pradimicin derivative. Antimicrob. Agents Chemother. 35: $2185 \sim 2190,1991$

8) Kamachi, H.; S. Iimura, S. Okuyama, H. Hoshi, S. Tamura, M. Shinoda, K. Saitoh, M. Konishi \& T. Oki: Synthesis and antifungal activities of pradimicin derivatives, modification at C4'-position. J. Antibiotics 45 : $1518 \sim 1525,1992$

9) Litchfield, J. T. \& F. Wilcoxon: A simplified method of evaluating dose-effect experiments. J. Pharmacol. Exp. Ther. 96: $99 \sim 113,1949$ 